\title{
Instrumento para Evaluar la Cohesión Social en Universidades Mexicanas: Resultados de la Validación Cultural UNIVECS-MX
}

\section{Instrument to Assess the Social Cohesion in Mexican Universities: Results of UNIVECS-MX Cultural Validation}

\author{
Carlos Sancho-Álvarez ${ }^{1,{ }^{*}}$, Mario Rueda Beltrán ${ }^{2}$, José González-Such ${ }^{1}$ y Jesús Miguel Jornet Meliá ${ }^{1}$ \\ ${ }^{1}$ Universitat de València, España \\ ${ }^{2}$ Universidad Nacional Autónoma de México, México
}

\section{DESCRIPTORES:}

Educación superior

Evaluación educativa

Validez cultural

Ítems test

Cohesión social

\begin{abstract}
RESUMEN:
El objetivo de este artículo es ofrecer un instrumento para evaluar la cohesión social en universidades mexicanas. A partir del modelo de evaluación UNIVECS validado en el contexto español, se realiza una adaptación y validación cultural en México; desarrollando un juicio de expertos con nueve profesionales del área de Antropología, Lingüística, Pedagogía, Psicología y Sociología, un jueceo con 51 docentes en educación superior, cuatro grupos de validación de reactivos entre tres universidades con 42 docentes, y un grupo de validación con diez estudiantes universitarios. Todo ello, en función de cuatro criterios de valoración cuantitativa y cualitativa: pertinencia, claridad, factibilidad y ausencia de sesgos lingüísticos, culturales, organizacionales y de género. A partir de los resultados y propuestas de mejora se obtiene un paquete de reactivos validados en torno a ocho dimensiones de análisis de la cohesión social en educación superior: Sostenibilidad, Bienestar Social, Inclusión, Autonomía Personal, Sentido de Pertenencia, Confianza, Movilidad Social y Responsabilidad Social. Los resultados posibilitan y concluyen un instrumento de evaluación pertinente, claro, factible y libre de sesgos. UNIVECS-MX se ofrece a las instituciones mexicanas para poder identificar en sus licenciaturas la cohesión social y poder realizar diagnósticos institucionales de cada dimensión propuesta, así como seguir realizando investigaciones socio-educativas.
\end{abstract}

\section{KEYWORDS: \\ Higher education \\ Educational \\ assessment \\ Cultural validity \\ Test items \\ Social cohesion}

\section{ABSTRACT:}

The objective of this article is to offer a system to assess the social cohesion in Mexican universities. Based on the UNIVECS evaluation model validated in the Spanish context, cultural adaptation and validation has been carried out in Mexico; developing an expert trial with 9 professionals in the area of Anthropology, Linguistics, Pedagogy, Psychology and Sociology, a judge with 51 teachers in higher education, 4 reagent validation groups between 3 universities with 42 teachers and a validation group with 10 students University students All this, based on four criteria of quantitative and qualitative assessment: relevance, clarity, feasibility and absence of linguistic, cultural, organizational and gender biases. From the results and proposals for improvement, a package of validated reagents is obtained based on eight dimensions of analysis of social cohesion in education: Sustainability, Social Welfare, Inclusion, Personal Autonomy, Sense of Belonging, Trust, Social Mobility and Responsibility Social. The results enable and conclude a pertinent, clear, feasible and bias-free evaluation system. UNIVECS-MX is offered to Mexican universities to be able to identify social cohesion in their university careers and make an institutional diagnosis of each proposed dimension.

CÓMO CITAR:

Sancho-Álvarez, C., Rueda, M., González-Such, J. y Jornet, J. M. (2021). Instrumento para evaluar la cohesión social en universidades mexicanas: resultados de la validación cultural UNIVECS-MX. Revista Iberoamericana de Evaluación Educativa, 14(1),115-133. https://doi.org/10.15366/riee2021.14.1.007

*Contacto: carlos.sancho@uv.es ISSN: 1989-0397

https://revistas.uam.es/riee 


\section{Introducción}

A partir del modelo de evaluación educativa para la Cohesión Social -CS- (Jornet, 2012) desarrollado y validado en universidades del contexto español como UNIVECS (Jornet et al., 2020), el cual plantea identificar la CS en las carreras universitarias, así como las aportaciones que realizan las instituciones a la misma (González-Such y Garrido, 2019; Jornet y Bakieva, 2019), presentamos el reto metodológico de adaptación socio-cultural del instrumento de evaluación en el contexto mexicano (Perales et al., 2019; Perales y Sancho-Álvarez, 2019), así como los resultados de la validación del mismo.

Para ello, debemos tener en cuenta un posicionamiento que integre la situación contextual donde se desarrolla la adaptación (a nivel local), así como a partir de la propia validación resultante avanzar sobre la internacionalización (a nivel global), reafirmando el compromiso de las universidades para la consecución de la cohesión social (Fëdorov et al., 2013). En este tipo de proceso evaluativo es imprescindible considerar las características y elementos diferenciales de los contextos hacia los cuales se plantea el proceso de adaptación y validación, dado que los aspectos culturales y de idioma pueden influir significativamente en la manera en que las personas dan sentido a sus experiencias y construyen significados (Bialystok, 2002; Wertsch, 1985). Por ello, es imprescindible un enfoque de validación cultural donde se realice el proceso de adaptación planteado, ya que la cultura permea en cualquier aspecto de evaluación (Solano-Flores et al., 2006; Wuttke, 2007). El punto de partida para ello es la definición de validez cultural de Solano-Flores y Milbourn (2016, p. 3): "La medida en que, durante todo el proceso de desarrollo de la evaluación, se toma en consideración que la experiencia cultural influye en la manera en que los estudiantes interpretan los ítems de una prueba”.

\section{Revisión de la literatura}

El instrumento de evaluación UNIVECS (Jornet et al., 2020) validado por el grupo de investigación Gem-Educo (2019) de la Universitat de València se fundamenta a partir de dos definiciones esenciales de CS. La primera del Consejo de Europa (2005), que atiende a "la capacidad de la sociedad para garantizar la sostenibilidad del bienestar de todos sus miembros, incluido el acceso equitativo a los recursos disponibles, la dignidad en la diversidad y la autonomía personal, colectiva y a la participación responsable" (p. 23).

La segunda con relación a la Comisión Económica para América Latina y el Caribe (CEPAL) que hace referencia a "la eficacia de los mecanismos instituidos de inclusión social como a los comportamientos y valoraciones de los sujetos que forman parte de la sociedad" (CEPAL, 2007, p. 19).

Esto implica que la cohesión social en las sociedades modernas no alude a la armonía social resultado de la existencia en un grupo de normas y valores únicos, sino al reconocimiento y valoración de la pluralidad de intereses e identidades existentes. Para ello se apela a un conjunto de valores definidos como universales y que están comprendidos en la dimensión prescriptiva: respeto a la diversidad cultural y a los derechos humanos, solidaridad social, igualdad social, participación cívica responsable para alcanzar metas comunes por medios democráticos, sentido de pertenencia a la Unión Europea, autonomía personal y la democracia como sistema idóneo de organización social (CONEVAL, 2015, p.24).

El objetivo que pretende es evaluar en qué medida se encuentran las carreras universitarias en torno a la CS y cómo contribuyen al desarrollo y transformación de la sociedad desde el concepto de CS (Jornet, 2012) para cada una de las siguientes dimensiones de medida validadas mediante una metodología de comités de expertos (González-Such y Sánchez-Delgado, 2019), tales como la Sostenibilidad -D1-, Bienestar Social -D2-, Inclusión -D3-, Autonomía Personal -D4-, Sentido de Pertenencia -D5-, Confianza -D6-, Movilidad Social -D7- y Participación -D8- (citadas en González-Such y Garrido, 2019, p. 835):

La dimensión 1 se define como "la capacidad de la carrera universitaria para formar a la comunidad universitaria en competencias que aseguren su desarrollo profesional y respondan a las necesidades presentes y futuras de la sociedad", y está formada por tres subdimensiones: (1.1) Institucional, (1.2) Profesorado y (1.3) Competencias Básicas. 
La dimensión 2 se define como "la capacidad de la carrera universitaria para mantener y mejorar la calidad de vida de su comunidad universitaria y de la sociedad, al garantizary promover el respeto de los derechos humanos", y está formada por una subdimensión: (2.1) Clima social y de aprendizaje.

La dimensión 3 se define como "la capacidad de la carrera universitaria para garantizar la equidad y atender la diversidad de necesidades, capacidades y particularidades de la comunidad universitaria, eliminando las barreras de acceso al aprendizaje, facilitando su participación e incrementando su rendimiento para su desarrollo personal y profesional", y está formada por tres subdimensiones: (3.1) Medidas metodológicas, (3.2) Medidas organizativasy (3.3) Consideración y respuesta a la diversidad.

La dimensión 4 se define como "la capacidad de la carrera universitaria para desarrollar entre la comunidad educativa la competencia para decidiry guiar su propio comportamiento", y está formada por tres subdimensiones: (4.1) Desarrollo de actitudes, (4.2) Planificación y ejecución de proyectos personales y profesionales y (4.3) Participación e implicación personal en proyectos colectivos.

La dimensión 5 se define como "la capacidad de la carrera universitaria para que las personas implicadas en la misma se identifiquen como grupo con sus valores y costumbres, reflejando un compromiso que implique la competencia para participar en decisiones dentro de su comunidad universitaria", y está formada por seis subdimensiones: (5.1) Responsabilidad y compromiso, (5.2) Respeto a los otros y símbolos de la institución, (5.3) Valores, (5.4) Emociones y sentimiento hacia la institución, (5.5) Aceptación de normas y (5.6) Hábitos y costumbres.

La dimensión 6 se define como "la capacidad de la carrera universitaria para generar su reconocimiento social a través de la honestidad, la benevolencia y las actitudes y valores compartidos" y está formada por cinco subdimensiones: (6.1) Reconocimiento social, (6.2) Honestidad, (6.3) Benevolencia, (6.4) Credibilidady (6.5) Integridad.

La dimensión 7 se define como "la capacidad de la carrera universitaria para promover y facilitar la mejora del estatus personal, profesional y social de la comunidad universitaria implicada" y está formada por dos subdimensiones: (7.1) Tasas y (7.2) Movilidad social.

La dimensión 8 se define como "la capacidad de la carrera universitaria para desarrollar entre la comunidad universitaria mecanismos formales y no formales de contribución/dinamización de la cohesión social", y está formada por tres subdimensiones: (8.1) Política, (8.2) Responsabilidad socialy (8.3) Académica.

Resulta imprescindible seguir conociendo y explorando cada contexto institucional porque únicamente desde las evidencias encontradas podemos mejorar los factores institucionales asociados al proceso educativo universitario, ya que, según Rueda y otros (2014), así como Luna y Rueda (2016), las evidencias de estudios sobre las condiciones universitarias en instituciones mexicanas pueden generar espacios de reflexión y debate en torno a los factores que pueden estar incidiendo en la calidad de la formación y en la práctica del aula universitaria.

\section{Método}

En este sentido, la metodología empleada para desarrollar la validación cultural considera diferentes fases y criterios de validación para poder potenciar sensibilidad hacia los diferentes elementos culturales del contexto, así como atención hacia las características diferenciales que surgen en el proceso de adaptación (Perales y Sancho-Álvarez, 2019).

La investigación se desarrolla a través de la técnica panel o juicio de expertos con profesionales de educación superior mexicana, ya que esta estrategia se utiliza en múltiples ámbitos de la evaluación para asegurar la validez de contenido en instrumentos de medición (Kitamura y Kitamura, 2000). Por ello, se ha convertido en muchas áreas de investigación como una herramienta esencial (Utkin, 2006): 
El juicio de expertos se define como una opinión informada de personas con trayectoria en el tema, que son reconocidas por otros como expertos cualificados en éste, y que pueden dar información, evidencia, juicios y valoraciones (Escobar-Pérez y Cuervo-Martínez, 2008, p. 29).

\subsection{Grupos de estudio}

Se desarrollaron tres periodos clave de investigación con diferentes grupos de estudio en cada contexto como juicio de expertos: 1) se realizó una adaptación lingüística y socio-cultural en la Universidad Nacional Autónoma de México-UNAM-, 2) se trabajó con diversos docentes de educación superior de todo México mediante un jueceo cuantitativo y 3) se analizaron los reactivos mediante jueceo cualitativo en tres universidades mexicanas de diferentes contextos geográficos: Universidad Autónoma de Baja California -zona norte-, Universidad Nacional Autónoma de México-zona centro-y la Universidad Autónoma de Yucatán -zona sur-.

\subsection{Periodo 1}

Inicialmente, se realiza una aproximación al contexto mexicano mediante visitas presenciales a la UNAM para observar sus instalaciones y estructura organizacional, así como para realizar búsquedas bibliográficas en sus repositorios y organizar reuniones in situ con docentes universitarios.

La adaptación del instrumento requiere en primer lugar su adaptación lingüística, ya que aunque entre ambos contextos -español y mexicano- se habla lengua castellana, es importante considerar que "los aspectos culturales asociados al idioma no siempre se consideran de manera formal" (Solano-Flores et al., 2006, p. 16). Por ello, se realiza un juicio de expertos por entrevista para la adaptación lingüística del instrumento de evaluación UNIVECS con nueve investigadores y docentes en activo de UNAM. Cada persona tiene una experiencia profesional de más de quince años, siendo profesionales a tiempo completo de diferentes ámbitos de conocimiento: Antropología, Lingüística, Pedagogía, Psicología y Sociología.

Tal y como plantean los autores Skjong y Wentworht (2000), la identificación de los participantes en el juicio de expertos es una parte crítica del proceso. Por lo que ha sido de especial importancia poder trabajar con profesionales con amplia experiencia en estas áreas, para poder realizar una primera adaptación lingüística y socio-cultural para el contexto mexicano a nivel cualitativo, en relación a la redacción y a la presentación de los indicadores del instrumento de evaluación UNIVECS.

Sin duda, todas estas precauciones iniciales son necesarias para trabajar la validez cultural del instrumento en el contexto planteado, dado que la cultura permea en cualquier aspecto de evaluación (Solano-Flores et al., 2006; Wuttke, 2007).

\subsection{Periodo 2}

En consecuencia, se realiza otro juicio de expertos por encuesta de valoración online con 51 profesionales de 21 instituciones mexicanas de educación superior procedentes de diversas áreas de conocimiento tales como Antropología, Artes, Biología, Economía, Filosofía, Historia, Humanidades, Ingenierías, Lingüística, Matemáticas, Pedagogía, Psicología, Sociología y Trabajo Social.

Algunos estudios anteriores de validación en relación con el modelo de CS (Bakieva et al., 2018; Sancho-Álvarez et al., 2017), aportan evidencias sobre la necesidad de trabajar con criterios de validación útiles para complementar la adaptación lingüística previa, centrando el procedimiento en la sensibilidad cultural sobre el proceso de validación (Solano-Flores y Milbourn, 2016). En este caso, el grupo de expertos analiza el instrumento de evaluación UNIVECS en función de sus dimensiones y reactivos, tomando en consideración diversos criterios de análisis con una escala de cuatro puntos (Totalmente en desacuerdo -1-, En desacuerdo -2-, De acuerdo -3-y Totalmente de acuerdo -4-), conjuntamente con apartados de espacio abierto para incluir recomendaciones o propuestas de mejora. Los criterios de análisis de fueron (adaptados de Perales et al., 2019):

- Pertinencia (P): Hasta qué punto la afirmación es relevante para medir la dimensión. En cada apartado aparece la definición de la dimensión que se pretende evaluar. 
- Claridad (C): Grado de claridad de la afirmación, si se entiende correctamente lo que se formula en el reactivo.

- Factibilidad (F): Hace referencia al grado en que el aspecto valorado por el reactivo puede ser cambiado a través de la formación universitaria.

- Ausencia de Sesgos (S): El reactivo, tal y como está definido, no presenta sesgos identificables en relación con algún colectivo por cuestiones culturales, lingüísticas, de género y/o organizacionales. Asimismo, en caso de identificar sesgos, se ruega indicar en espacio abierto una propuesta de redacción alternativa que permita evitar el sesgo señalado.

\subsection{Periodo 3}

Por último, se desarrollaron un total de seis grupos focales presenciales de validación de reactivos en diferentes momentos de investigación y grupos de estudio, tal y como se muestra en la descripción siguiente -véase Cuadro 1-, donde también se trabajan los criterios anteriormente expuestos; sin embargo, en este caso únicamente de manera cualitativa y en función del consenso grupal para tomar decisiones sobre los reactivos.

Cuadro 1.

Grupos de estudio y número participantes por universidad y estado

\begin{tabular}{cccc}
\hline Grupo & Universidad & Participantes & Estado \\
\hline 1 & UABC & 8 docentes & Baja California \\
2 & UABC & 12 docentes & Baja California \\
1 & UNAM & 9 docentes & Ciudad de México \\
2 & UNAM & 12 docentes & Ciudad de México \\
3 & UNAM & 10 estudiantes & Ciudad de México \\
1 & UADY & 6 docentes & Yucatán \\
\hline
\end{tabular}

Nota. Elaboración propia.

Según Gable y Wolf (1993), Grant y Davis (1997), y Lynn (1986) (citados en McGartland et al. 2003) la cantidad adecuada para el juicio de expertos se sitúa entre dos y veinte participantes, mientras que Hyrkäs et al. (2003) sugieren que diez participantes brindarían una estimación confiable; aunque el número de grupos y participantes es similar por contexto; sin embargo, no resulta proporcional ya que por motivos de tiempo y disponibilidad no fue posible.

\section{Resultados}

Los análisis descriptivos y estadísticos de los datos cuantitativos se han procesado mediante el apoyo del software SPSS v.2O, realizando análisis descriptivos de las respuestas, análisis de consistencia y nivel de acuerdo sobre las valoraciones cuantitativas de los participantes en el juicio de expertos. En este sentido, se calcula el coeficiente de $W$ Kendall y el coeficiente de correlación Intra-clase para comprobar la concordancia y consistencia del acuerdo en las valoraciones, ya que son considerados los análisis más adecuados para escalas ordinales de valoración con cuatro puntos (Escobar-Pérez y Cuervo-Martínez, 2008); utilizadas en cada criterio de análisis y valoración para evitar el error de tendencia central y la deseabilidad social en las respuestas (Baka y Figgou, 2012; Johns, 2005; Velez y Ashworth, 2007).

\subsection{Análisis descriptivo del jueceo}

A continuación se presentan los resultados del jueceo con las valoraciones emitidas por los expertos en educación superior mexicana sobre cada reactivo propuesto. 
Los datos se estructuran en torno a las dimensiones del instrumento de evaluación UNIVECS (Sostenibilidad -D1-, Bienestar Social -D2-, Inclusión -D3-, Autonomía Personal -D4-, Sentido de Pertenencia -D5-, Confianza -D6-, Movilidad Social -D7- y Responsabilidad Social -D8-), así como se pueden observar los análisis descriptivos en función de cada criterio de análisis (Pertinencia-P-, Claridad-C-, Factibilidad -F-y Ausencia de Sesgos -S-) con base a la escala de cuatro opciones de respuesta: 1-Muy en desacuerdo-, 2-En desacuerdo-, 3-De acuerdo- y 4-Muy de acuerdo-.

En la D1 se observan puntuaciones superiores a 3,4 puntos en todos los criterios y reactivos (Figura 1).

Figura 1

Valoraciones D1: Sostenibilidad

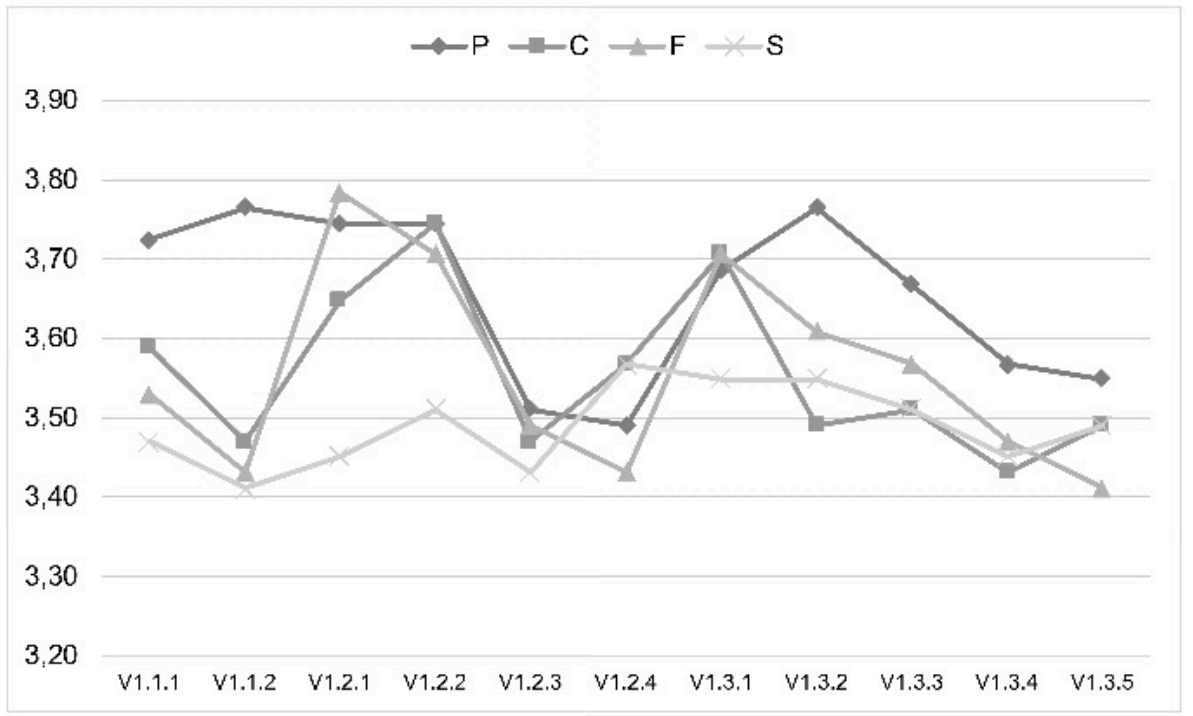

Nota. Elaboración propia.

En las valoraciones emitidas para la D2, se observa que las puntuaciones han sido superiores a 3,35 puntos en todos los casos (Figura 2).

Figura 2

Valoraciones sobre D2: Bienestar Social

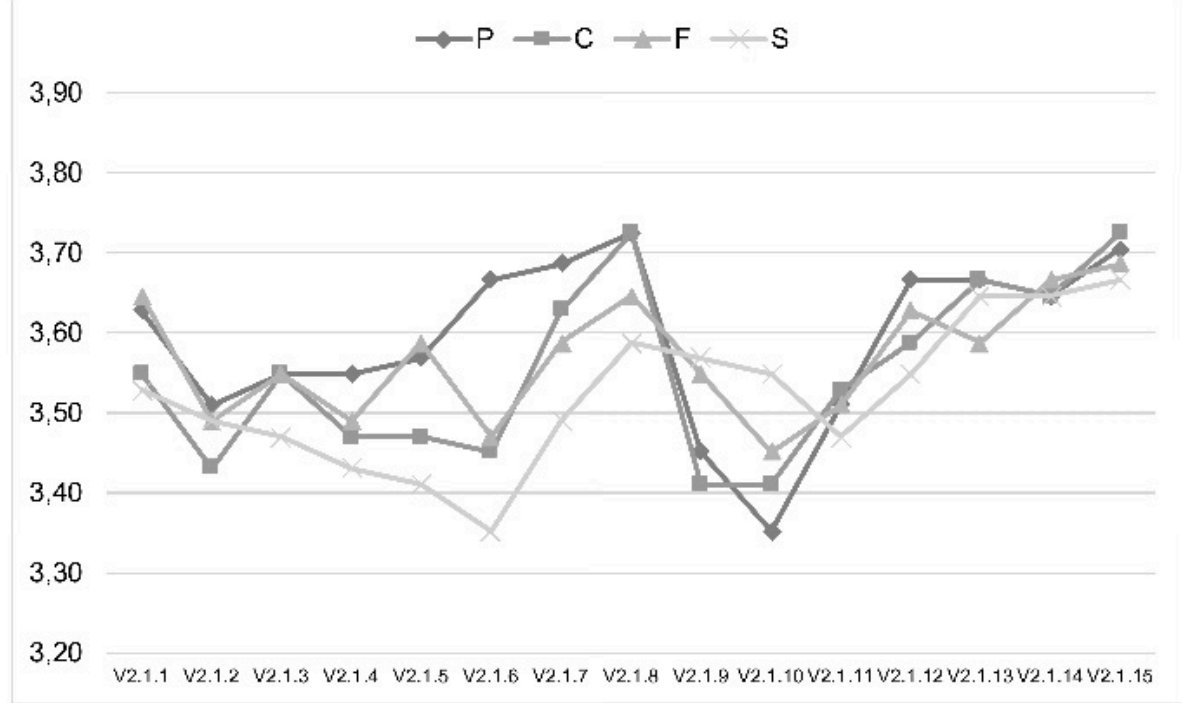

Nota. Elaboración propia. 
Después del análisis descriptivo de las valoraciones sobre la D3, todos los resultados han superado los 3,2 puntos en situación de análisis (Figura 3).

Figura 3

Valoraciones sobre D3: Inclusión

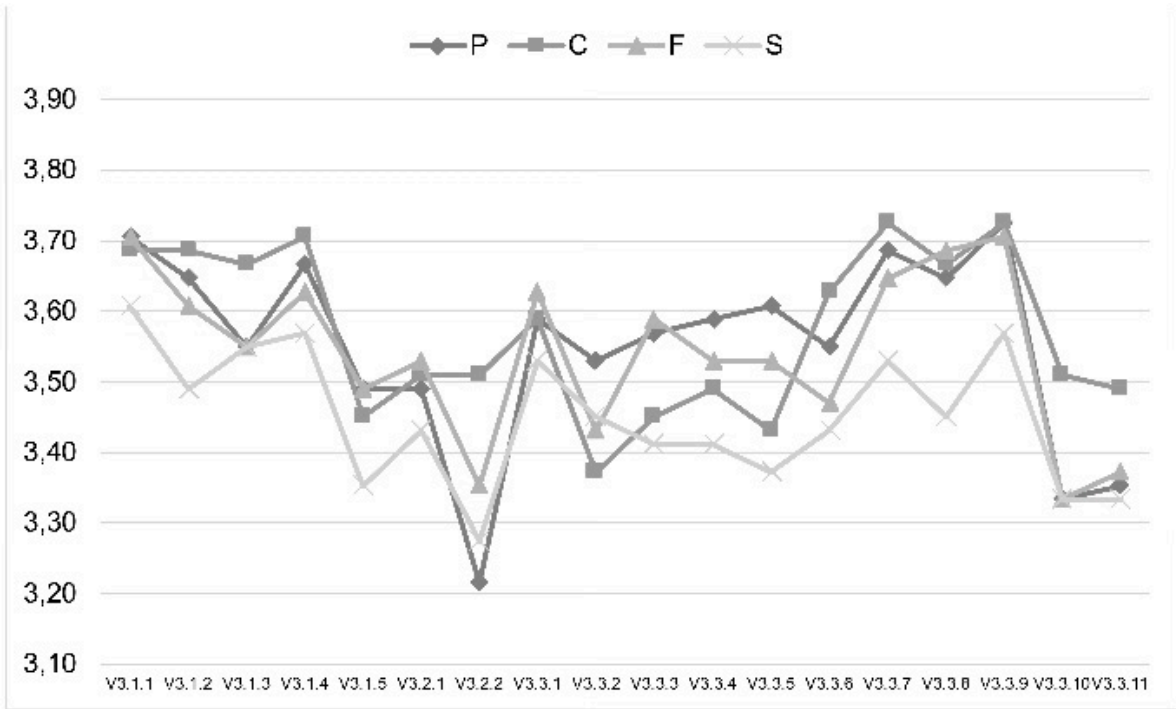

Nota. Elaboración propia.

Todas las valoraciones sobre la D4 son superiores a 3,4 puntos (Figura 4).

Figura 4

Valoraciones sobre D4: Autonomía Personal

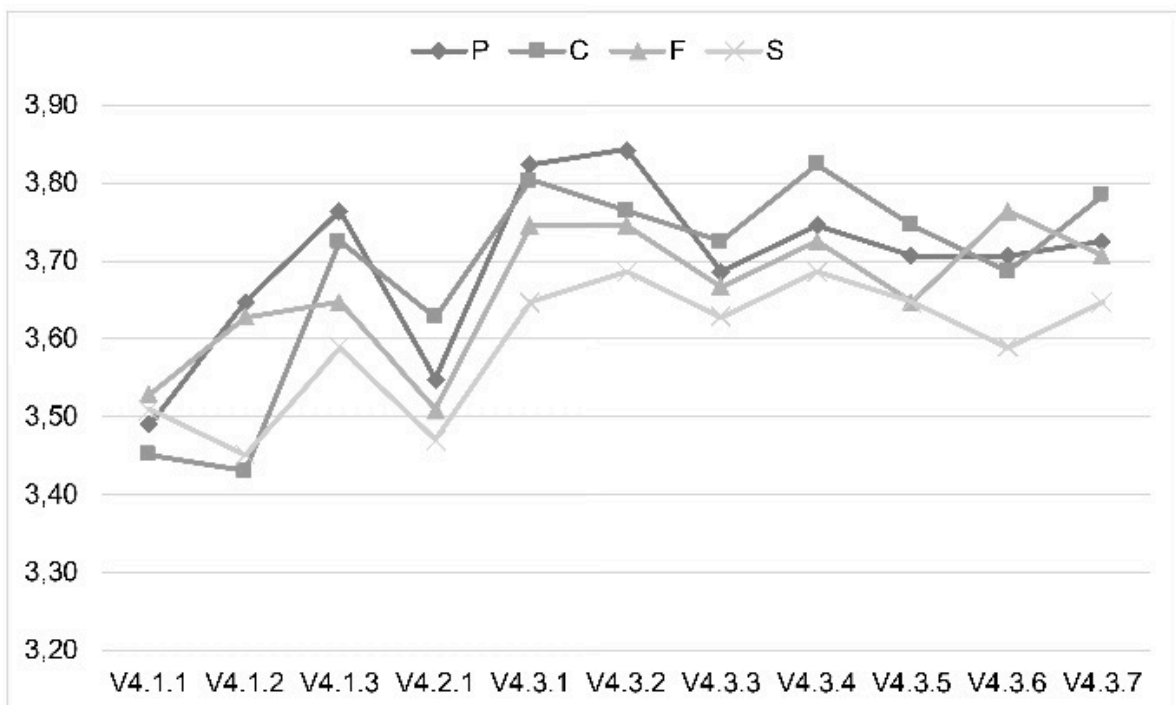

Nota. Elaboración propia.

En el caso de las puntuaciones emitidas para la D5, todos los resultados indican que han superado en cualquier aspecto los 3,35 puntos (Figura 5). 


\section{Figura 5}

Valoraciones sobre D5: Sentido de Pertenencia

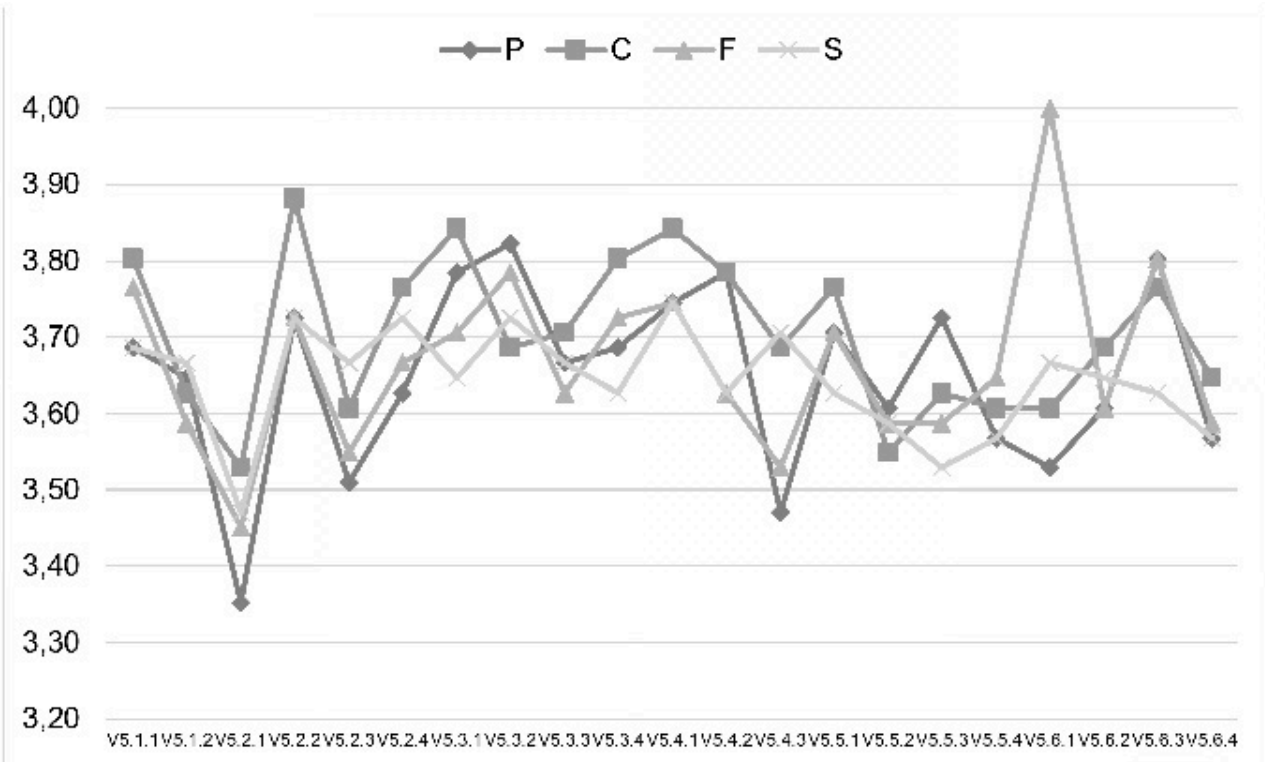

Nota. Elaboración propia.

En la D6 se observan valores superiores a 3,2 puntos en todos los casos (Figura 6).

Figura 6

Valoraciones sobre D6: Confianza

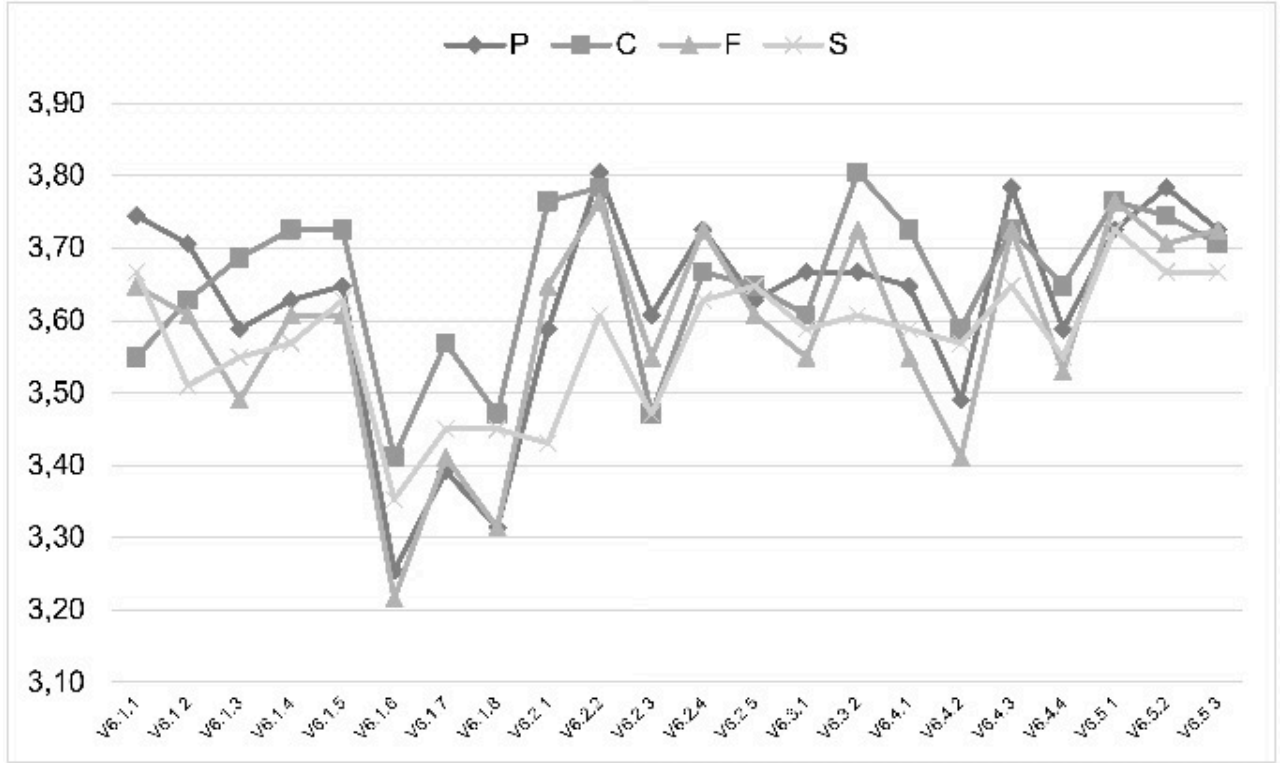

Nota. Elaboración propia.

En cuanto a los datos emitidos para valorar la $\mathrm{D} 7$ observamos que en todos los reactivos y criterios analizados se superan los 3,55 puntos (Figura 7). 
Figura 7

Valoraciones sobre D7: Movilidad Social

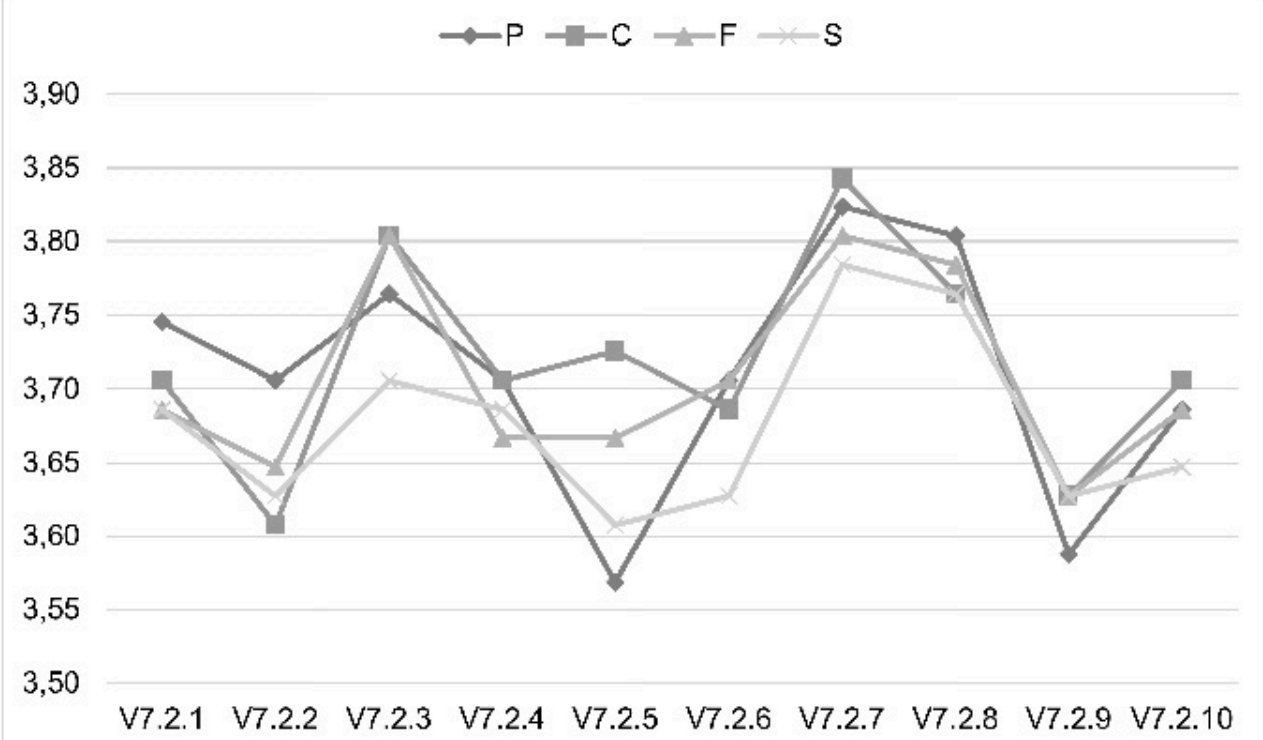

Nota. Elaboración propia.

Después del análisis descriptivo sobre las valoraciones de la D8, los resultados en todos los casos son superiores a los 3,15 puntos (Figura 8).

Figura 8

Valoraciones sobre D8: Responsabilidad Social

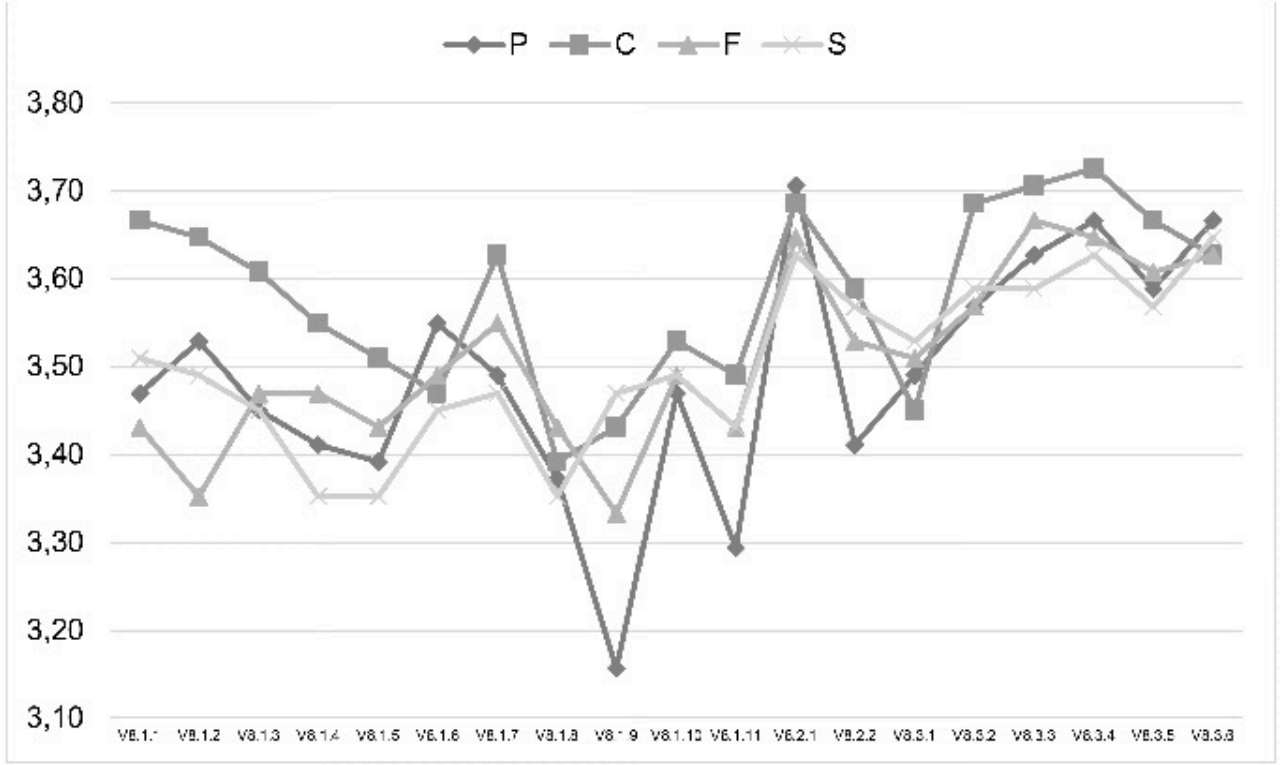

Nota. Elaboración propia.

Asimismo, después de observar las valoraciones descriptivas de manera individual para todas las dimensiones de análisis y criterios de validación para cada reactivo, se presentan los totales descriptivos por dimensiones y criterios de manera global (Figura 9). 
Figura 9

Valoraciones sobre totales en cada dimensión por criterios de análisis

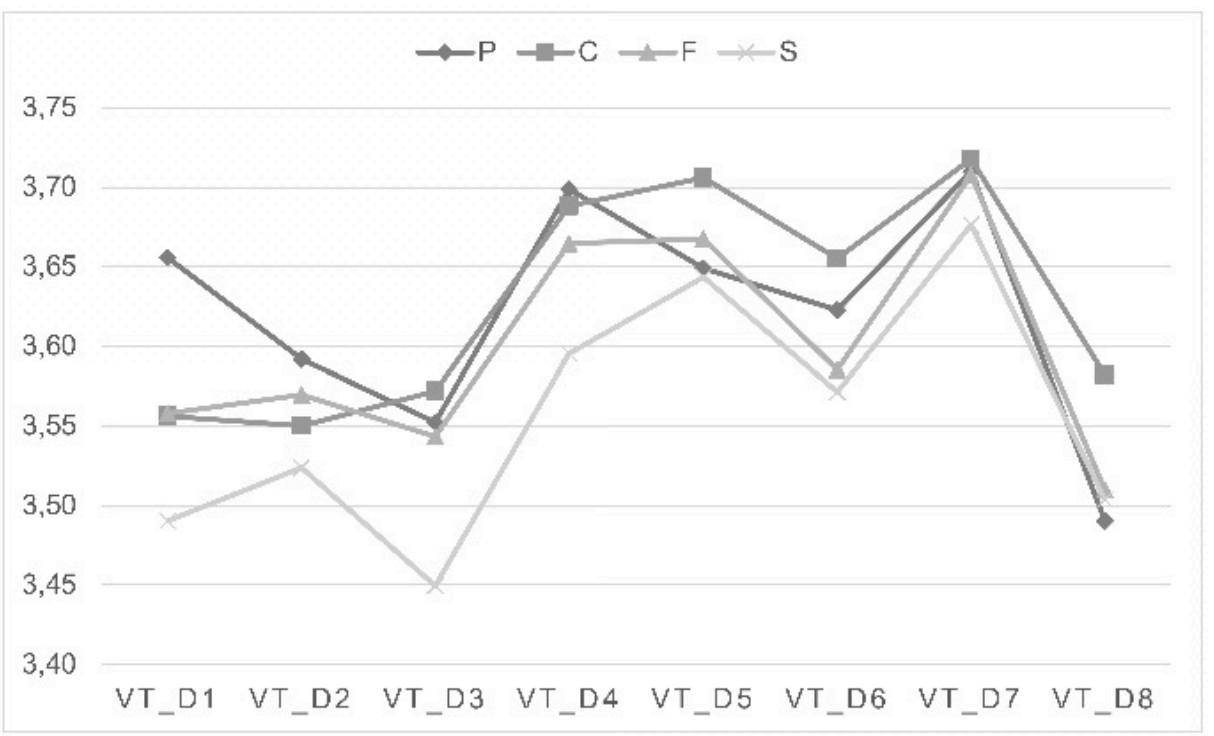

Nota. Elaboración propia.

Se puede observar en la Figura 10 que todas las dimensiones con relación a cada criterio superan las puntuaciones de 3, 45 puntos, considerado en el rango. Sin embargo, como podemos observar en los datos totales, encontramos algunas diferencias entre las opiniones sobre los criterios valorados, ya que cierta tendencia implica al criterio de Ausencia de Sesgos (S) menores puntuaciones en todas las dimensiones; algo que se tendrá que seguir analizando con el siguiente tipo de análisis para comprobar el acuerdo estadístico inter-jueces, ya que observar los datos de manera descriptiva y calcular el porcentaje de acuerdo resulta una medida insuficiente porque no incluye el acuerdo esperado por el azar (Jakobsson y Westergren, 2005).

\section{Grado concordancia inter-jueces}

Después de observar los análisis descriptivos en las valoraciones de cada dimensión y criterios analizados, se han realizado los siguientes análisis estadísticos para comprobar el grado de acuerdo sobre las valoraciones emitidas por los jueces.

Cuadro 2

Concordancia acuerdo inter-jueces por dimensiones y criterios de análisis

\begin{tabular}{|c|c|c|c|c|c|c|c|c|}
\hline \multirow[b]{2}{*}{$D^{1}$} & \multicolumn{2}{|c|}{ Pertinencia } & \multicolumn{2}{|c|}{ Claridad } & \multirow{2}{*}{$\begin{array}{c}\text { Factibilidad } \\
W-K^{2}\end{array}$} & \multicolumn{3}{|c|}{ Ausencia de Sesgos } \\
\hline & $W-K^{2}$ & $I-C^{4}$ & $W-K^{2}$ & $I-C^{4}$ & & $I-C^{4}$ & $W-K W-K^{2}$ & $I-C^{4}$ \\
\hline 1 &, $520^{*}$ & ,647* & ,393* & ,395 & ,479* & ,709* &, $603^{*}$ &,- 922 \\
\hline 2 &, $551^{*}$ & ,593* &, $447^{*}$ &, 525 & ,490* & 227 &, $585^{*}$ & ,430 \\
\hline 3 &, $463^{*}$ &, $575^{*}$ & ,440* & ,486 &, $482^{*}$ & ,522 & ,493* & ,162 \\
\hline 4 &, $507^{\star}$ &, 576 &, $485^{*}$ &, $754^{*}$ &, $517^{*}$ & ,308 &, $660^{*}$ & ,452 \\
\hline 5 &, $507^{*}$ & ,603* & ,469* &, $547^{*}$ &, $539 *$ &, $672^{*}$ & ,693* & 247 \\
\hline 6 &, $518^{*}$ &, $722^{*}$ & ,490* &, $541^{*}$ & ,489* & ,699* &, $622^{*}$ & ,387 \\
\hline 7 &, $576^{*}$ & ,317 &, $540^{*}$ & ,230 &, $549^{*}$ &,- 055 &, $651^{*}$ &,- 002 \\
\hline 8 & ,648* &, $618^{*}$ &, $693^{*}$ &, $519 *$ & ,683* & ,385 & ,790* &, $529^{*}$ \\
\hline
\end{tabular}

Notas: ${ }^{1}$ Dimensión ${ }^{2}$ W Kendall ${ }^{3}$ Significaciónasintótica (bilateral) ${ }^{4}$ CorrelaciónIntra-clase ${ }^{*}$ Acuerdoestadísticamente significativo Elaboración propia. 


\section{Discusión}

En cuanto a los datos cuantitativos, de manera descriptiva se obtienen puntuaciones adecuadas en todos los reactivos de manera individual, así como entre los totales de las dimensiones analizadas; obteniendo una valoración total en Pertinencia de 3,62 ( $\sigma 0,41)$, en Claridad de 3,62 ( $\sigma 0,35)$, en cuanto a Factibilidad de 3,60 $(\sigma 0,43)$ y en Ausencia de Sesgos una puntuación media de 3,55 ( $\sigma 0,50)$. Asimismo, los datos muestran que en todas las dimensiones y criterios existe acuerdo estadísticamente significativo en cuanto al coeficiente de Kendall. Sin embargo, únicamente en el criterio de Pertinencia el coeficiente de correlación Intra-clase reafirma el consenso inter-jueces a modo de consistencia interna. Algo que confirma que la Claridad, Factibilidad y Ausencia de Sesgos se debe seguir analizando de manera diferencial por reactivos en cuanto a las valoraciones cualitativas aportadas de los jueces en los apartados de observaciones con espacios abiertos, en busca de relaciones.

Sin embargo, el criterio que menos puntuación obtiene en todas las dimensiones es el de Ausencia de Sesgos (S); algo que resulta necesario analizar junto a las valoraciones de los juicios de expertos de validación cualitativa, ya que es un aspecto muy importante a tener en cuenta para evitar sesgos en los reactivos y su formulación adecuada.

Por ello, se analizan los datos cualitativos y las recomendaciones encontradas en los apartados de observaciones junto al juicio de expertos y los datos descriptivos y estadísticos, así como las puntuaciones individuales de los reactivos y se deciden eliminar ciertos ítems defectuosos, así como mantener y/o cambiar la formulación y redacción de otros ítems, de acuerdo a las propuestas de mejora de los expertos participantes; ya que según afirman Voutilainen y Liukkonen (1995, citados en Hyrkäs et al., 2003) si un 80\% de los expertos han estado de acuerdo con la validez de un grupo de reactivos éstos pueden ser incorporados al instrumento de evaluación o de lo contrario se deben revisar.

Asimismo, se vuelven a revisar los reactivos de acuerdo con las aportaciones de los comités de validación presenciales del periodo tres, junto con los criterios de valoración y el porcentaje anteriormente citado como medida de calidad para la incorporación de ítems. También, a partir de las recomendaciones de los expertos en los diferentes grupos focales de validación de reactivos, se concluye que tal y como están planteados los ítems pueden servir para cualquier universidad mexicana o institución d educación superior ya que no presentan sesgos organizacionales ni tampoco tendencias que puedan excluir su aplicación en ninguna organización al respecto de su formulación.

Finalmente, se analizan todas las propuestas de mejora de cada grupo de validación, las recomendaciones, datos cualitativos y cuantitativos para concluir con el instrumento de evaluación UNIVECS adaptado al contexto mexicano -UNIVECS-MX- (veáse Cuadro 3) para evaluar la cohesión social en estudiantes y docentes universitarios; resultado que ha sido posible gracias a todas las aportaciones de las audiencias implicadas en los diferentes momentos de investigación y en relación a las fases metodológicas anteriormente citadas entre todas las universidades participantes en el estudio de validación cultural en México.

Cuadro 3

UNIVECS-MX Sistema para evaluar la cohesión social en universidades mexicanas

\begin{tabular}{lll}
\hline D & SD & REACTIVOS \\
\hline & 111 Mi licenciatura tiene asignaturas que corresponden a su perfil de conocimiento (D) \\
& 112 Mi licenciatura tiene un procedimiento para actualizar el diseño curricular que respondan a \\
& las necesidades presentes y futuras de la sociedad (D) \\
& 121 Mi licenciatura tiene asignaturas que se imparten por docentes con experiencia (DE) \\
& 122 Mi licenciatura tiene docentes actualizados en el dominio de su área de conocimiento (DE) \\
& 124 Mi licenciatura tiene un número de estudiantes que permite atender a la diversidad del \\
& grupo-clase (D) (MI: 3.2) \\
& 133 La formación en mi licenciatura desarrolla habilidades para resolver problemas en el área de \\
& estudio (DE) \\
& 134 En mi licenciatura se promueve la reflexión profesional respecto a temas relevantes del área \\
& de conocimiento (DE) \\
& 135 La formación en mi licenciatura posibilita la difusión del conocimiento del área de estudio \\
& (DE) \\
& 136 La formación en mi licenciatura desarrolla habilidades de aprendizaje autónomo necesarias \\
& para realizar estudios de posgrado (D)
\end{tabular}


211 En mi licenciatura se promueve la comunicación entre estudiantes (DE)

212 En mi licenciatura se promueve la comunicación entre docentes (D)

213 En mi licenciatura se promueve la colaboración entre estudiantes (DE)

214 En mi licenciatura se promueve la colaboración entre docentes (D)

215 En mi licenciatura se promueve la cohesión grupal a través de diferentes estrategias (DE)

216 En mi licenciatura se promueve la convivencia entre las personas (DE)

217 En mi facultad se promueven relaciones respetuosas entre las personas (DE)

$219 \mathrm{Mi}$ licenciatura tiene uno o varios proyectos compartidos entre asignaturas (D)

$2110 \mathrm{Mi}$ facultad tiene recursos (personal, espacios y tiempos) para la colaboración entre docentes (D)

$2111 \mathrm{Mi}$ facultad tiene condiciones de trabajo que facilitan la colaboración entre estudiantes (E)

2113 En mi universidad se protegen los derechos humanos (DE)

$2114 \mathrm{Mi}$ universidad ofrece mecanismos para mediar en conflictos surgidos entre las personas de la comunidad universitaria (DE)

2115 Las personas de mi universidad se relacionan de manera respetuosa (DE)

$2116 \mathrm{Mi}$ universidad ofrece protocolos ante conflictos/delitos para garantizar los derechos universitarios (DE)

311 Mi licenciatura tiene docentes actualizados en metodologías didácticas sobre atención a la diversidad (D)

313 La formación en mi licenciatura ofrece retroalimentación a partir de mis resultados de evaluación (E)

314 En mi licenciatura hay aulas donde se trabaja de forma colaborativa (DE)

315 En mi licenciatura se promueve la atención a la diversidad en las aulas mediante estrategias didácticas (D)

316 Mi facultad tiene apoyos pertinentes a estudiantes/docentes con algún tipo de discapacidad (DE)

3.2 .

$322 \mathrm{Mi}$ facultad tiene recursos suficientes para responder a la diversidad de estudiantes (D) $323 \mathrm{Mi}$ universidad ofrece un área destinada a la atención de personas con discapacidad (DE)

$332 \mathrm{Mi}$ licenciatura tiene criterios de evaluación justos para la diversidad de estudiantes (DE) 333 En mi licenciatura se promueve la sensibilización en relación con la discapacidad (DE) 334 En mi licenciatura se promueve la sensibilización en relación con la diversidad socio-cultural (DE)

335 En mi licenciatura se promueve la sensibilización en relación con la diversidad sexual (DE) 336 Mi universidad es accesible y está adaptada a las necesidades de estudiantes/docentes con discapacidad (DE)

3.3. 337 En mi universidad la diversidad entre estudiantes enriquece la enseñanza y el aprendizaje (DE)

338 En mi universidad la atención a la diversidad en las aulas es posible (D)

339 Las personas de mi universidad creen que la inclusión educativa es necesaria para alcanzar la inclusión social (DE)

3310 En mi licenciatura se trabaja con materiales didácticos que contemplan la perspectiva de género $(\mathrm{DE})$

3311 En mi licenciatura se trabaja con materiales didácticos que contemplan la diversidad cultural y lingüística (DE) 
411 La formación en mi licenciatura posibilita el reconocimiento de responsabilidades indivi-

4.1 duales al realizar tareas (DE)

413 La formación en mi licenciatura posibilita la adquisición de la responsabilidad como un valor imprescindible de crecimiento personal (E)

$4.2 \quad 424$ En mi licenciatura se promueve la participación en problemáticas relativas a la universidad (DE)

431 La formación en mi licenciatura posibilita la resolución de problemas grupales asumiendo la responsabilidad personal (E)

432 La formación en mi licenciatura posibilita la construcción de un criterio personal para aplicar en trabajos grupales (E)

433 En mi licenciatura se promueve la participación en proyectos de investigación e innovación de la universidad (DE)

434 En mi licenciatura se promueve la concienciación personal en iniciativas sociales y solidarias (DE) (MI: 8.2)

435 En mi licenciatura se promueve la participación en proyectos colectivos de la universidad (jornadas, extra-curriculares, etc) (DE) (MI: 1.3)

436 En mi licenciatura se promueve la participación en proyectos de la universidad que trascienden las obligaciones académicas (DE) (MI:5.1.3)

437 En mi licenciatura se promueve la información de actividades de carácter social o humanitario que ofrece la universidad (DE) (MI: 8.2; 5.7)

511 La formación en mi licenciatura promueve el compromiso para alcanzar los objetivos académicos (DE) (MI: 6.5)

512 Mi universidad promueve que seamos corresponsables del funcionamiento de la licenciatura (DE)

521 Las personas de mi universidad utilizan accesorios (plumas, ropa, etc) con el logotipo de la institución (DE)

522 Las personas de mi universidad comunican fuera de la institución que pertenecen a ella (DE)

523 En mi universidad se atribuye el mismo valor a todas las iniciativas (académicas, culturales, deportivas, etc) (DE)

531 Las personas de mi universidad conocen los valores de la institución (DE) (MI: 6.5)

533 Las personas de mi universidad hacen visibles y proyectan los valores de la institución en su comunidad (DE)

5.3 534 Las personas de mi universidad contribuyen a la difusión social de los valores institucionales (DE)

535 Las personas de mi universidad se identifican con los valores institucionales (DE) (MI: 6.5)

$541 \mathrm{Mi}$ licenciatura tiene estudiantes que manifiestan orgullo por estudiarla (E)

5.4 544 Las personas de mi universidad se identifican con la cultura universitaria de la institución (DE)

$551 \mathrm{Mi}$ universidad ofrece una legislación institucional justa (DE)

$552 \mathrm{En} \mathrm{mi} \mathrm{universidad} \mathrm{la} \mathrm{transparencia} \mathrm{es} \mathrm{una} \mathrm{característica} \mathrm{institucional} \mathrm{(DE)}$

5.5 553 Las personas de mi universidad se esfuerzan por cumplir la legislación universitaria (DE) 554 En mi universidad el cumplimiento de la legislación universitaria mejora el funcionamiento de la licenciatura (DE)

561 Mi universidad facilita que se permanezca de forma voluntaria en las instalaciones más tiempo del que comprende el horario de clases (DE) $563 \mathrm{Mi}$ universidad ofrece actividades complementarias (extensión cultural, deportivas, idiomas...) en sus instalaciones (DE)

$564 \mathrm{Mi}$ universidad ofrece un ambiente universitario que favorece el hábito de trabajo/estudio (DE)

5.7 (MIs: Sub-dimensiones 8.1; 8.2; 8.3) 
612 En mi licenciatura se promueve una visión positiva de la profesión a nivel social (DE)

613 La formación en mi licenciatura facilita la inserción laboral (DE)

6.1 ticas, intercambios...) (E)

616 En mi licenciatura se promueve información sobre las posibles opciones laborales (E)

$618 \mathrm{Mi}$ licenciatura tiene reconocimiento social (E)

$6110 \mathrm{Mi}$ licenciatura tiene demanda en su matrícula (E)

622 La formación en mi licenciatura posibilita el desarrollo ético de la profesión (DE)

$6.2623 \mathrm{Mi}$ licenciatura tiene docentes que se comportan de manera honesta y congruente con el estudiante (E)

6 625 La formación en mi licenciatura posibilita la identificación de limitaciones personales (E)

6.3 631 Las personas de mi universidad hacen que prevalezca el interés común sobre el propio (DE) 632 En mi universidad se escuchan las necesidades de la comunidad universitaria (DE)

$641 \mathrm{En}$ mi universidad las autoridades universitarias brindan apoyo cuando se requiere (DE) $643 \mathrm{Mi}$ licenciatura tiene estudiantes que confían en la calidad y fortaleza del plan de estudios (E) $644 \mathrm{La}$ formación en mi licenciatura responde a los intereses y expectativas del estudiante (DE) $652 \mathrm{Mi}$ universidad es coherente entre lo que dice y lo que realmente hace (misión, visión, valores, etc) (DE)

\subsection{Indicadores tasas}

722 Mi universidad ofrece información sobre convocatorias y acceso a becas (estudios, manutención, excelencia, etc) (DE)

724 En mi licenciatura se promueve la colaboración con diferentes colectivos socioprofesionales: colegio profesional, asociaciones profesionales, centros de prácticas, centros de investigación, etc (DE) (MI: 1.1)

726 En mi universidad se generan oportunidades de promoción para reconocer y potenciar habilidades personales y profesionales (D) (MI: 4.1)

727 La formación en mi licenciatura mejora la capacidad de tomar decisiones (E) (MI: 4.1) zaciones para el desarrollo de proyectos compartidos (D) (MI: 1.1)

7210 Mi universidad promueve que sus docentes participen en actividades de formación continua (D) (MI: 1.2)

7211 En mi facultad se promueven iniciativas de formación y desarrollo personal entre la comunidad educativa (DE) (MI: 4.2)

7212 La formación en mi licenciatura perfecciona cualidades personales y habilidades sociales

(E) (MI: 4.2)

7213 La formación en mi licenciatura posibilita la planificación y gestión de un proyecto propio

(E) (MI: 4.2) 
811 Mi universidad ofrece información sobre los miembros de los consejos de representación (DE) (MI: 5.1)

$813 \mathrm{Mi}$ universidad ofrece información sobre las responsabilidades de ser miembro en consejos de representación $(\mathrm{DE})$

815 En mi universidad las personas representantes se preocupan por conocer la opinión de la comunidad universitaria (DE)

817 Mi universidad ofrece información sobre convocatorias para ser representante en algún consejo (DE)

818 En mi universidad la participación en los consejos de representación mejora las situaciones de injusticia (DE)

8110 Las personas de mi universidad tienen interés por participar en consejos o grupos de representación (DE) (MI: 5.7)

8111 Las personas de mi universidad participan en las elecciones de consejos de representación (DE) (MI: 5.7; 5.1)

823 En mi facultad se promueven actividades extracurriculares para fomentar actitudes cívicas y humanitarias (DE)

824 Las personas de mi universidad participan voluntariamente en diferentes organizaciones no lucrativas de carácter social (DE) (MI: 5.7)

831 En mi licenciatura se promueve la participación en las actividades de clase (E)

$832 \mathrm{Mi}$ licenciatura tiene horarios de clases que facilitan la asistencia a actividades extracurriculares (E)

$834 \mathrm{En} \mathrm{mi} \mathrm{facultad} \mathrm{se} \mathrm{promueven} \mathrm{actividades} \mathrm{entre} \mathrm{asignaturas,} \mathrm{semestres} \mathrm{y} \mathrm{facultades} \mathrm{(DE)}$

8.3 835 En mi universidad la participación en actividades extracurriculares es relevante (DE)

836 En mi facultad se promueven actividades que se organizan desde la licenciatura (D) (MI: 5.7)

837 En mi facultad se promueven eventos no universitarios que se consideran interesantes para la formación académica (DE) (MI: 5.7)

Nota. Se indica al final de cada reactivo la audiencia de respuesta: D-docente- y/o E-estudiante-, así como los Multi-Indicadores (MI) que están validados para dos dimensiones simultáneas y son intercambiables.

\section{Conclusiones}

La propuesta de cuidar la validez cultural en la adaptación del contenido y el formato de los ítems (Solano-Flores y Milbourn, 2016) exige en la adaptación a otros contextos un especial esfuerzo y dedicación para que la interpretación de la prueba sea pertinente, clara, útil y libre de sesgos lingüísticos, culturales, organizacionales y de género. Para ello, se ha realizado un minucioso proceso de validación cultural del instrumento de evaluación para la cohesión social UNIVECS-MX con estudiantes y docentes universitarios, con la finalidad de ofrecer a las instituciones de educación superior mexicana una propuesta útil como instrumento de mejora. Así como seguir aportando evidencias de validación para perfilar una propuesta adecuada al contexto investigado.

En este sentido, se realizaron diversas reuniones y conferencias informativas de manera presencial en cada universidad con estudiantes y docentes para explicar el proyecto, así como la prospectiva y líneas de investigación a corto, medio y largo plazo.

En este sentido, una vez validado el sistema UNIVECS-MX a continuación se procede a su aplicación con estudiantes y docentes en diferentes carreras universitarias de las universidades participantes, a través de una aplicación de metodología mixta por medio de grupos de discusión y encuestas que se desarrolla 
como una investigación de complementariedad metodológica mediante un acercamiento tanto cuantitativo como cualitativo (Bericat, 1998; Dellinger y Leech, 2007). En este caso, se presentarán los resultados de estas fases posteriores en otros estudios para realizar una adecuada y completa difusión de resultados (Sales y Folkman, 2001).

La difusión de resultado es un deber ético en investigación (Salazar et al., 2018) y como estudio socialmente comprometido (Murillo e Hidalgo, 2017) debemos devolver el máximo de información posible a las audiencias implicadas para poder establecer vías de trabajo hacia la mejora de los contextos investigados, así como ofrecer los resultados como herramientas útiles de trabajo.

Finalmente, en cuanto a la prospectiva de análisis junto con ambos extremos de investigación -validación de constructo y de escala de evaluación-, se podrá adaptar el instrumento de manera adecuada al contexto participante, así como seguir obteniendo evidencias de validación adecuadas sobre los resultados, pudiendo dar respuesta a la adaptación completa en estudios posteriores con el objetivo de encontrar argumentos orientativos y detallados hacia posibles modificaciones y mejoras (Mérida et al., 2015).

Este enfoque metodológico se ha establecido como el marco de referencia hacia el desarrollo de modelos de evaluación en los últimos años (Fisher y Jackson, 2011). Al respecto se enfatiza la validación de constructo como un elemento de bondad sustantivo para cualquier instrumento de evaluación educativa, siendo de vital importancia las evidencias de validación como parte prioritaria sobre la validación de la escala y su interpretación junto con otras variables (Jornet y González-Such, 2009). Con todo, este tipo de estudios pueden mejorar la validez en los procesos de evaluación educativa de la investigación aplicada, a través de la implementación de una evaluación sumativa y/o formativa o como valoración/diagnóstico institucional, ya que se han diseñado y construido junto con los principales implicados (Jornet et al., 2020).

\section{Agradecimientos}

Agradecimientos a toda la Red Iberoamericana de Investigadores sobre la Evaluación de la Docencia-RIIEDpor su apoyo y colaboración en esta investigación.

Este trabajo se ha realizado en el marco del proyecto I+D+I Validación de un sistema de evaluación de titulaciones universitarias basado en un modelo de Cohesión Social UNIVECS (EDU2016-78065R) financiado por el Ministerio de Economía, Industria y Competitividad de España, y apoyado con fondos FEDER de la Unión Europea, y del proyecto postdoctoral Validación cultural en universidades mexicanas del instrumento de evaluación para la Cohesión Social UNIVECS (INV18-01-19-06) financiado por el Subprograma Atracció de Talent 2018 de la Universitat de Valéncia.

\section{Referencias}

Baka, A. y Figgou, L. (2012). 'Neither agree, nor disagree': A critical analysis of the middle answer category in voting advice applications. International Journal Governance, 5(3), 244-263. https://doi.org/10.1504/IJEG.2012.051306

Bakieva, M., Jornet, J. M., González-Such, J. y Leyva, Y. (2018). Colegialidad docente: Evidencias de validación a partir del análisis realizado por comités de expertos acerca del instrumento para autoevaluación docente en España y México. Estudios sobre Educación, 34, 99-127. https://doi.org/10.15581/004.34.99-127

Bericat, E. (1998). La integración de los métodos cuantitativo y cualitativo en la investigación social. Significado y medida. Ariel.

Bialystok, E. (2002). Cognitive processes of L2 users. En V. J. Cook (Ed.), Portraits of the L2 user (pp. 145-165). BMultilingual Matters.

CEPAL. (2007). Un sistema de indicadores para el seguimiento de la cohesión social en América Latina. CEPAL-EUROsociAL.

CONEVAL. (2015). Cohesión social: balance conceptual y propuesta teórico metodológica en México. CONEVAL. 
Consejo de Europa. (2005). Elaboration concertée des indicateurs de la cohésion sociale - Guide méthodologique. Consejo de Europa.

Dellinger, A. y Leech, N. (2007). Toward a unified validation framework in mixed methods research. Journal of Mixed Methods Research, 14, 309-332. https://doi.org/10.1177/1558689807306147

Escobar-Pérez, J. y Cuervo-Martínez, A. (2008). Validez de contenido y juicio de expertos: una aproximación a su utilización. Avances en Medición, 6, 27-36.

Fëdorov, A. N., González-Such, J. y Jornet, J. M. (2013). Internacionalización de la educación superior y cohesión social: algunos elementos de reflexión. En J. Gacel-Ávila, y N. Orellana, N. (Coords), Educación Superior: gestión, innovación e internacionalización (p.161-194). JPM Ediciones.

Fhiser, W. P. y Jackson, A. (2011). Integrating qualitative and quantitative research approaches via the phenomenological method. International Journal of Multiple Research Approaches, 5(1), 85-99.

https://doi.org/10.5172/mra.2011.5.1.89

González-Such, J. y Garrido, P. (2019). Lineamientos para el diseño de estrategias de implementación de la evaluación en el modelo UNIVECS [Comunicación invitada]. XIX Congreso Internacional de Investigación Educativa AIDIPE2019, Madrid, España.

González-Such, J. y Sánchez-Delgado, P. (2019). Soluciones metodológicas para la selección y diseño de medidas de percepción para el modelo UNIVECS. [Comunicación invitada]. XIX Congreso Internacional de Investigación Educativa AIDIPE2019, Madrid, España.

Hyrkäs, K., Appelqvist-Schmidlechner, K y Oksa, L. (2003). Validating an instrument for clinical supervision using an expert panel. International Journal of nursing studies, 40(6), 619-625. https://doi.org/10.1016/S0020-7489(03)00036-1

Jakobsson, U. y Westergren, A. (2005). Statistical methods for assessing agreement for ordinal data. Scandinavian Journal of Caring Sciences, 19(4), 427-431. https://doi.org/10.1111/j.1471-6712.2005.00368.x

Johns, R. (2005). One size doesn't fit all: selecting response scales for attitude items. Journal of Elections, Public Opinion and Parties, 15(2), 237-264. https://doi.org/10.1080/13689880500178849

Jornet, J. M. (2012). Dimensiones Docentes y Cohesión Social: Reflexiones desde la Evaluación. Revista Iberoamericana de Evaluación Educativa, 5(1), 349-362.

Jornet, J. M. y Bakieva, M. (2019, junio). Diseño de indicadores simples y complejos para la evaluación de entrada, proceso, product y context para el modelo UNIVECS. Comunicación invitada en el XIX Congreso Internacional de Investigación Educativa AIDIPE2019, Madrid, España.

Jornet, J. M., Bakieva, M. y Sánchez-Delgado, P. (2020). La Cohesión Social como objetivo de la educación: ¿Podemos especificar un modelo de calidad para realizar la evaluación de Sistemas Educativos? Fronteiras: Journal of Social, Technological and Environmental Science (en prensa).

Jornet, J. M. y González-Such, J. (2009). Evaluación criterial: Determinación de estándares de interpretación (EE) para pruebas de rendimiento educativo. Estudios sobre Educación, 16, 103-123.

Jornet, J. M., Perales Montolío, M. J. y González-Such, J. (2020). El concepto de validez de los procesos de evaluación de la docencia. Revista Española de Pedagogía, 78(276), 233-252.

https://doi.org/10.22550/REP78-2-2020-01

Kitamura, T. y Kitamura, F. (2000). Reliability of clinical judgment of patients' competency to give informed consent: A case vignette study. Psychiatry and Clinical Neurosciences, 54(2), 245-247.

https://doi.org/10.1046/j.1440-1819.2000.00665.x

Luna, E. y Rueda, M. (2017). Experiencias de evaluación de la docencia en Iberoamérica. Universidad Autónoma de Baja California.

McGartland, D. Berg, M., Tebb, S. S., Lee, E. S. y Rauch, S. (2003). Objectifying content validity: Conducting a content validity study in social work research. Social Work Research, 27(2), 94-104.

Mérida, R., Serrano, A. y Tabernero, C. (2015). Diseño y validación de un cuestionario para la evaluación de la autoestima en la infancia. Revista de Investigación Educativa, 33(1), 149-162. https://doi.org/10.6018/rie.33.1.182391 
Murillo, F. J. e Hidalgo, N. (2017). Hacia una investigación educativa socialmente comprometida. Revista Iberoamericana de Evaluación Educativa, 10(2), 5-8.

Perales, M. J. y Sancho-Álvarez, C. (2019, junio). Estrategias metodológicas para la adaptación a otros contextos socio-culturales: la internacionalización del modelo UNIVECS. Comunicación invitada en el XIX Congreso Internacional de Investigación Educativa AIDIPE2019, Madrid, España.

Perales, M. J., Sancho-Álvarez, C. y González-Such, J. (2019, julio). Orientaciones metodológicas para la validación cultural del modelo UNIVECS. Comunicación invitada en el $8^{\circ}$ Congreso Ibero-Americano en Investigación Cualitativa CIAIQ2019, Lisboa, Portugal.

Rueda, M., Canales, A., Leyva, Y. E. y Luna, E. (2014). Condiciones contextuales para el desarrollo de la práctica docente. Revista Iberoamericana de Evaluación Educativa, 7(2), 171-183.

Salazar, M. B., Icaza, M. F. y Alejo, O. J. (2018). La importancia de la ética en la investigación. Revista Universidad y Sociedad, 10(1), 305-311.

Sales, B. D. y Folkman, S. (2001). Ethics in research with human participants. APA.

Sancho-Álvarez, C., Jornet, J. M. y González-Such, J. (2017). Una aproximación al valor social subjetivo de la educación en Iberoamérica Evidencias de validez entre Argentina, España y República Dominicana. Perfiles Educativos, 34(157), 14-33.

Skjong, R. y Wentworth, B. (2000). Expert judgement and risk perception. http://research.dnv.com/skj/Papers/SkjWen.pdf

Solano-Flores, G. y Milbourn, T. (2016). Capacidad evaluativa, validez cultural y validez consecuencial en PISA. Revista Electrónica de Investigación y Evaluación Educativa, 22(1), M12. https://doi.org/10.7203/relieve.22.1.8281

Solano-Flores, G., Contreras-Niño, L. A. y Backhoff, E. (2006). Traducción y adaptación de pruebas: Lecciones aprendidas y recomendaciones para países participantes en TIMSS, PISA y otras comparaciones internacionales. Revista Electrónica de Investigación Educativa, 8(2).

Utkin, L. V. (2006). A method for processing the unreliable expert judgments about parameters of probability distributions. European Journal of Operational Research, 175(1), 385-398.

Velez, P. y Ashworth, S. D. (2007). The impact of item readability on the endorsment of the midpoint reponse in surveys. Survey Research Methods, 1(2), 69-74.

Wertsch, J. V. (1985). Vygotsky and the social formation of mind. Harvard University Press.

Wuttke, J. (2007). Uncertainties and bias in PISA. En S. T. Hopmann, G. Brinek, and M. Retzl (Eds.), According to PISA - Does PISA keep what it promises? (pp. 241-263). LIT Verlag.

\section{Breve CV de los autores}

\section{Carlos Sancho-Álvarez}

Doctor en Educación, Máster en Psicopedagogía Social y Licenciado en Pedagogía por la Universitat de València. Maestro por la Universidad de Alcalá de Henares. Ha sido educador y formador en todas las etapas del sistema educativo español, así como ha impartido docencia en diferentes universidades sobre medición educativa, evaluación de programas, educación inclusiva y metodologías de investigación. Ha realizado estancias de investigación en universidades de Argentina, México y USA. Miembro del grupo de innovación docente Innovamide, del grupo de investigación Gem-Educo (UV) y de la RIIED. Actualmente es investigador postdoctoral del departamento MIDE (UV) y desarrolla la validación cultural en México del sistema de evaluación UNIVECS-MX. Email: carlos.sancho@uv.es

ORCI ID: https://orcid.org/0000-0001-9489-2502 


\section{Mario Rueda Beltrán}

Doctor en CC de la Educación por la Universidad de París VIII, Francia, y Licenciado en Psicología por la Universidad Nacional Autónoma de México. Investigador Nacional desde 1987 del SNI de CONACyT y Miembro de la Academia Mexicana de Ciencias desde 2008. Ha impartido docencia universitaria en psicología y pedagogía en UNAM desde 1972, así como capacitaciones sobre didáctica, evaluación y metodología de investigación en diversas universidades nacionales e internacionales. Ha sido presidente del INEE, director del IISUE y ha promocionado la investigación educativa en México desde el COMIE. Ha coordinado diversos proyectos de investigación sobre evaluación de la docencia a nivel nacional como internacional. Actualmente es coordinador de la RIIED e investigador titular del IISUE-UNAM. Sus líneas de investigación giran en torno a la evaluación de la docencia y las condiciones institucionales de la educación superior.Email: mariorb@unam.mx

ORCID ID: https://orcid.org/0000-0001-5004-4915

\section{José González-Such}

Doctor en CC de la Educación y Licenciado en Pedagogía por la Universitat de València. Ha impartido docencia sobre estadística aplicada, medición educativa y metodologías de investigación en CC Sociales/ Educación. Profesor del máster de Política, Gestión y Dirección de Centros Educativos, máster en Psicopedagogía y director del máster de Educación Especial (UV). Ha coordinado diversos proyectos de innovación educativa y evaluación del profesorado. Actualmente es catedrático del departamento MIDE (UV), coordinador del grupo Innovamide y miembro del grupo de investigación Gem-Educo (UV), así como de la RIIED. Sus líneas de trabajo actuales se centran en medición y evaluación educativas, evaluación del profesorado, innovación educativa y la validación del sistema UNIVECS para evaluar la cohesión social en universidades. Email: jose.gonzalez@uv.es

ORCID ID: https://orcid.org/0000-0001-9086-6446

\section{Jesús Miguel Jornet Meliá}

Doctor en CC de la Educación y Licenciado en Filosofía y CC de la Educación (Psicología) por la Universitat de València. Ha impartido docencia en métodos de investigación, medición educativa, análisis de datos y evaluación educativa en diversos grados, másters y doctorados en España, Uruguay, Perú y República Dominicana. Ha colaborado como asesor del INEE y en la Unidad de Evaluación Educativa de la UABC, así como participa en diferentes consejos científico-académicos y proyectos a nivel nacional e internacional. Ha coordinado diversos proyectos I+D+I (España) sobre evaluación educativa. Actualmente es catedrático del departamento MIDE (UV), coordinador del grupo de investigación Gem-Educo (UV) y miembro de la RIIED. Sus líneas de trabajo actuales se centran en el diseño de instrumentos de evaluación educativa y la validación del sistema UNIVECS para evaluar la cohesión social en universidades. Email: jornet@uv.es

ORCID ID: https://orcid.org/0000-0001-6905-497X 